\title{
VIABILIDADE OVARIANA COM O USO DO EXTRATO DE SEMENTE DE UVA: UMA REVISÃO SISTEMÁTICA
}

Andre Petrolini ${ }^{1}$

\begin{abstract}
RESUMO
O objetivo foi examinar sistematicamente a literatura para verificar a influência do extrato de semente de uva como fator antioxidante no tratamento da saúde ovariana, or meio de busca e seleção da literatura em artigos publicados em língua inglesa nas bases de dados Lilacs, PubMed, SciELO, Science Direct/Elvesier e Google Acadêmico. Descritores utilizados foram grape (uva) "AND" seed (semente) "AND” ovary (ovário) "OR" ovarian (ovariano), sendo localizados 9.100 artigos. Realizadas leituras de títulos, resumos e textos completos, 03 manuscritos preencheram os critérios de inclusão. $\mathrm{O}$ uso do extrato de procianidina de semente de uva e do extrato proantocianidina de semente de uva obtiveram redução de ROS, quanto ao uso do extrato de semente de uva foi observado redução de LDL. O extrato de semente de uva pode ser benéfico para a viabilidade da saúde ovariana, pois causa homeostase a respeito do estresse oxidativo, no entanto, faz-se necessário estudos mais aprofundados.
\end{abstract}

Palavras-chaves: antioxidante, fertilidade, resíduos, uva, ovário

\section{OVARIAN VIABILITY WITH THE USE OF GRAPE SEED EXTRACT: A SYSTEMATIC REVIEW}

\begin{abstract}
The objective was to review systematically the literature to verify the influence of grape seed extract as an antioxidant factor in the treatment of ovarian health. by search and selection of literature in articles published in English in the Lilacs, PubMed, SciELO, Science Direct / Elvesier and Google Scholar databases. Descriptors used were grape (grape) "AND" seed (seed) "AND" ovary (ovary) "OR" ovarian (ovarian), with 9,100 articles found. Readings of titles, abstracts and full texts were performed, 03 manuscripts fulfilled the inclusion criteria. s: The use of grape seed procyanidin extract and grape seed proanthocyanidin extract obtained a reduction in ROS, while LDL reduction was observed in the use of grape seed extract. As conclusion grape seed extract can be beneficial for the viability of ovarian health, as it causes homeostasis regarding oxidative stress, however, further studies are necessary.
\end{abstract}

Keywords: antioxidant, fertility, waste, grape, ovary

\section{VIABILIDAD OVÁRICA CON EXTRACTO DE SEMILLA DE UVA: UNA REVISIÓN SISTEMÁTICA}

\section{RESUMEN}

El objetivo fue analisar sistemáticamente la literatura para verificar la influencia del extracto de semilla de uva como factor antioxidante en el tratamiento de la salud ovárica. Por medio de la búsqueda y selección de literatura en artículos publicados en inglés en las bases de datos

\footnotetext{
${ }^{1}$ Universidade Federal do Vale do São Francisco. Correspondência. andre.petrolini@ univasf.edu.br
} 
Lilacs, PubMed, SciELO, Science Direct / Elvesier y Google Scholar. Los descriptores utilizados fueron uva (uva) "AND" semilla (semilla) "AND" ovario (ovario) "OR" ovárico (ovario), con 9,100 artículos encontrados. Se realizaron lecturas de títulos, resúmenes y textos completos, 03 manuscritos cumplieron los criterios de inclusión. El uso de extracto de procianidina de semilla de uva y extracto de proantocianidina de semilla de uva obtuvo una reducción en ROS, mientras que se observó una reducción de LDL en el uso de extracto de semilla de uva. El extracto de semilla de uva puede ser beneficioso para la viabilidad de la salud ovárica, ya que causa homeostasis con respecto al estrés oxidativo, sin embargo, se necesitan más estudios.

Palabras clave: antioxidante, fertilidad, residuos, uva, ovário

\section{INTRODUÇÃO}

Com o aumento na produção de uvas, a indústria vinícola produz cada vez mais resíduos de biomassa que, do ponto de vista ambiental, pode ser utilizado para uma finalidade benéfica ao ser humano e ao meio ambiente (1). Reutilizá-lo resolveria, pelo menos em parte, os problemas de armazenagem e eliminação da biomassa das vinícolas. A importância em reutilizar o bagaço deve-se principalmente ao fato de seu conteúdo ser rico em lipídios, compostos bioativos como vitamina $\mathrm{E}$ e outros com atividades fitoterápicas, importantes para as indústrias alimentícia, farmacêutica e de cosméticos (2).

As sementes da uva são a parte do resíduo agroindustrial da vitivinicultura e da indústria dos sucos, extensivamente estudada devido seu alto potencial antioxidante (3). Os efeitos benéficos dos extratos obtidos da semente incluem a modulação da expressão de enzimas antioxidantes (4), a proteção contra danos oxidativos neurais de ratos (5), aterosclerose em hamster (6), inibição de metástase em tumor mamário (7) e alguns efeitos anti-inflamatórios (8).

As sementes da uva constituem uma proporção considerável do bagaço, no valor de 38 a $52 \%$ com base na matéria seca (9). De acordo com outros estudos (10), a composição de sementes de uva são, basicamente, $40 \%$ de fibra, $16 \%$ de óleo essencial, $11 \%$ de proteína e $7 \%$ de compostos fenólicos complexos como taninos e outras substâncias como açúcares e minerais.

Produtos de uvas como a farinha de semente de uva, têm sido utilizados para aplicações em alimentos por suas atividades antioxidantes, retardando a oxidação lipídica. Mas também, são consideradas por suas propriedades antimicrobianas contra bactérias mesófilas aeróbias e bactérias produtoras de ácido láctico (11). O extrato de bagaço de uva foi capaz de proteger a atividade de enzimas hepáticas (12), além de apresentar diversas atividades farmacológicas, como propriedades contra a oxidação das lipoproteínas de baixa densidade, prevenção de doenças cardiovasculares, redução do colesterol, dilatação dos vasos sanguíneos, entre outras (13).

Os produtos industrializados de uva contém a substância resveratrol (14), a qual possui capacidade antioxidante, agindo na célula folicular. Em estudo que utilizou $2 \mu \mathrm{M}$ de resveratrol adicionado ao meio de cultivo in vitro de tecido ovariano de ovino, observou-se a promoção da ativação de folículos primordiais, estimulação da proliferação celular e redução da fragmentação de DNA dos folículos (15).

Diante do exposto, o objetivo desse estudo foi examinar sistematicamente a literatura, para verificar a influência do extrato de semente de uva como fator antioxidante no tratamento da saúde ovariana. 


\section{MÉTODO}

Nesta revisão, buscaram-se artigos nas bases eletrônicas LILACS (Literatura LatinoAmericana e do Caribe em Ciências da Saúde), PUBMED (National Library of Medicine and The National Institute of Health), SciELO (Scientific Eletronic Library Online), Science Direct/Elvesier e Google Acadêmico, publicados em língua inglesa, entre janeiro de 2010 e abril de 2020. Na estratégia de busca, os descritores utilizados foram grape (uva) "AND" seed (semente) "AND" ovary (ovário) "OR" ovarian (ovariano), com o filtro para localizar as palavras somente descritas no título do referido artigo em inglês, verificadas anteriormente no DeCS/bvs (Descritores em Ciências da Saúde/ Biblioteca Virtual em Saúde), sendo localizados 9.100 artigos. (Quadro1)

Por meio deste procedimento de busca, identificaram-se os artigos que atenderam aos seguintes critérios de inclusão (Figura.1): pesquisas experimentais que abordassem os efeitos do extrato e derivados de semente de uva em ovários, publicados no idioma inglês e que tivessem no título as três palavras "grape", "seed" e "ovary/ovarian". Foram excluídos artigos com outro desenho metodológico, resultados com outras substâncias associadas a uva, como também, os artigos em duplicidade.

Após a primeira análise, com avaliação dos títulos, 07 artigos (LILACS=00; PUBMED=02; SciELO=00; Science Direct/Elsevier=00; e, Google Acadêmico=05) foram considerados elegíveis para a segunda fase desta revisão, que consistiu na leitura dos resumos. Após avaliação dos resumos, os estudos que pareciam preencher os critérios de inclusão foram lidos na íntegra. Nesta etapa, a revisão foi efetuada independentemente por dois pesquisadores. Ao final, 03 artigos atenderam a todos os critérios de inclusão.

$\mathrm{Na}$ avaliação dos artigos, foram observados os seguintes aspectos:

- amostra animal;

- utilização de derivados de extrato de semente de uva;

- aspectos éticos (menção a aprovação no comitê de ética).

Quadro 1. Principais fontes de informações primárias

\begin{tabular}{|c|c|c|}
\hline Fonte & Endereço & Características \\
\hline PubMed & https://www.ncbi.nlm.nih.gov/pubmed/ & Norte-americana \\
\hline LILACS & https://lilacs.bvsalud.org/ & América Latina \\
\hline Science Direct & https://www.sciencedirect.com/ & Europa \\
\hline Scielo & http://www.scielo.br/?lng=pt & Brasileiro \\
\hline Google Acadêmico & https://scholar.google.com.br/?hl=pt & Muito abrangente \\
\hline
\end{tabular}

Nota: LILACS (Literatura Latino-Americana e do Caribe em Ciências da Saúde), PUBMED (National Library of Medicine and The National Institute of Health), SciELO (Scientific Electronic Library Online) 


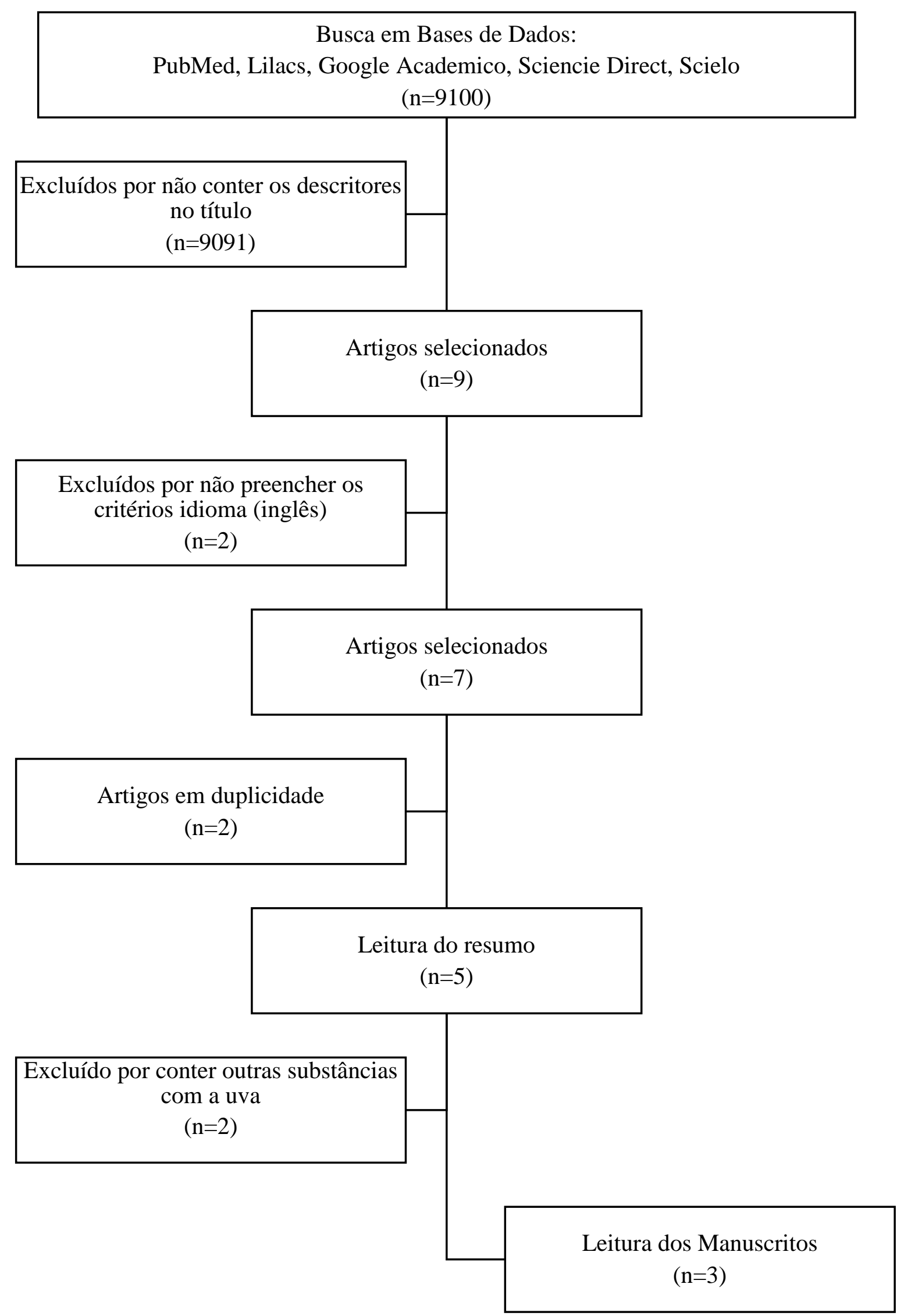

Figura1. Fluxograma de revisão 


\section{RESULTADOS}

No quadro 2, são apresentadas informações gerais sobre os 03 estudos incluídos, estes publicados de 2017 a 2019, como utilização de espécies animais e substâncias distintas. Observou se nos estudos que utilizaram, respectivamente, extrato de procianidina de semente de uva e extrato proantocianidina de semente de uva houve redução de ROS, já o estudo que fez uso do extrato de semente de uva ocorreu redução de LDL.

Quadro 2. Estudos sobre uso do extrato de semente de uva em ovários encontrados no título do artigo

\begin{tabular}{|c|c|c|c|}
\hline Autor/Ano do estudo & Espécie & Substância & Achados Relevantes \\
\hline Zhang et al., 2019 & suíno & $\begin{array}{l}\text { extrato de procianidina B2 de } \\
\text { semente de uva }\end{array}$ & redução ROS \\
\hline Liu et al., 2018 & ave & $\begin{array}{l}\text { extrato de proantocianidina de } \\
\text { semente de uva }\end{array}$ & redução ROS \\
\hline $\begin{array}{l}\text { Salmabadi et al., } \\
2017\end{array}$ & murino & extrato de semente de uva & redução de LDL \\
\hline
\end{tabular}

Nota: POCS -Síndrome do Ovário Policístico, LDL - Lipoproteína de baixa densidade, ROS Espécie Reativa de Oxigênio

\section{DISCUSSÃO}

O estresse oxidativo ocorre quando existe a perda do equilíbrio entre produção e eliminação de espécies reativas de oxigênio (ROS), utilizando como exemplo o oxigênio, que durante o transporte de elétrons na mitocôndria pode ser reduzido parcialmente gerando ROS, tais como ânion superóxido $\left(\mathrm{O}_{2}^{-}\right)$, peróxido de hidrogênio $\left(\mathrm{H}_{2} \mathrm{O}_{2}\right)$ e radical hidroxila $\left(\mathrm{OH}^{-}\right)$, neste caso podem ocorrer danos ao DNA, RNA, lipídios e proteínas. Além de fragmentação do DNA, as ROS podem causar o mal funcionamento do sistema de reparo do DNA, contribuindo para o desenvolvimento de doenças como o câncer (16).

Com uma grande quantidade de espécies reativas de oxigênio para produzir tecidos esteroidogênicos, o ovário processa a reação de conversão de colesterol em pregnenolona, catalisada pela enzima desmolase do complexo enzimático citocromo $\mathrm{P} 450$, produzindo um elevado número de elétrons (17). No ovário, as ROS, podem contribuir para o envelhecimento e surgimento de doenças (18).

Uma maneira eficaz para a redução de danos oxidativos no ovário é a suplementação por meio de antioxidantes (19). O extrato de proantocianidina derivado de sementes de uva (GSPE) é um potente antioxidante e eficaz eliminador de radicais livres (20), que alivia os danos oxidativos nos testículos e fígado de camundongos ao ativar a sinalização Nrf2 (21), alivia a apoptose do GC (célula da granulosa) induzida pelo estresse oxidativo, evocando uma resposta autofágica (22); como também, diminui a apoptose durante a cultura in vitro e a geração de ROS em vários tipos de células (23). Estudos anteriores enfatizam que o uso do extrato de semente de uva, além de conter uma grande quantidade de antioxidante, podem trazer benefícios para os ovários evitando seu envelhecimento precoce, ocasionado uma prole mais saudável de acordo com as evidências de estudos abaixo (Quadro 2). 
No estudo, foi investigado o potencial do procianidina 2 do extrato de semente de uva (GSP) para aliviar a apoptose das células da granulosa (GC), induzida por estresse oxidativo em ovários de suínas. Os resultados indicaram que as atividades das enzimas antioxidantes foram evidenciadas e os níveis de ROS e MDA (malondialdeído) aumentaram, significativamente, no tecido ovariano tratado com Diquat quando comparado ao controle, todavia, essas tendências foram atenuadas pelo complemento simultâneo do GSP. A capacidade antioxidante dos tecidos ovarianos diminuiu após o tratamento com Diquat e o GSP poderia inibir essa redução (24). O Diquat é um indutor do estresse oxidativo in vivo (25).

Desta forma considera-se o uso de extrato de semente de uva ou suas substâncias presentes como um potencial alimento antioxidante para a redução de danos causados pela oxidação celular, ocasionado em um processo degenerativo.

Distinto estudo (26) do quadro 2, utilizaram blocos corticais ovarianos de galinhas, tratadas com D-gal (2,5 mg / mL), GSPE (extrato de proantocianidina de semente de uva) $(5 \mu \mathrm{g} / \mathrm{mL})$ e D-gal + GSPE e observam que o GSPE reduziu significativamente os níveis de ROS, MDA e $\mathrm{H}_{2} \mathrm{O}_{2}$ (peróxido de hidrogênio) em ovários em envelhecimento induzidos por D-gal, e em ovários por envelhecimento natural in vitro. Ocorreu também uma redução nas atividades das enzimas antioxidantes, nos níveis de oxidantes e nos níveis de expressão de genes antioxidantes nos tecidos ovarianos envelhecidos induzidos por D-gal e tecidos ovarianos envelhecidos naturais foram inibidos após a suplementação de GSPE. Como conclusão, o GSPE auxiliou na proteção do estresse oxidativo nos ovários em envelhecimento das galinhas, e o modelo de envelhecimento ovariano induzido por D-gal representou um método prospectivo para o rastreamento farmacológico de antienvelhecimento.

Outro estudo (27) relacionado ao quadro 2, apresentou o tratamento com extrato de semente de uva, tendo como alvo a síndrome dos ovários policísticos (POCS), distúrbio endócrino que provoca a anovulação e consequente subfertilidade, teve como objeto de estudo o efeito do extrato de semente de uva (GSE) no triglicerídeo (TG), colesterol total (CT), lipoproteína de colesterol de alta densidade (HDL-C), lipoproteína de colesterol de baixa densidade (LDL-C) e interleucina-6 (IL-6) em ratos Wistar com PCOS. Após a intervenção foi coletado sangue para mensurar TG, medido pela glicerol-3-fosfato oxidase-peroxidase (GPO-PAP), TC pelo colesterol oxidase-peroxidase (CHOD-PAP) e HDL-C pelo método de sedimentação, LDL-C pelo cálculo de Friedewald e IL-6 pelo método ELISA.

Em todos os grupos experimentais, uma redução significativa da gordura visceral foi óbvia em comparação com o grupo PCOS controle. Os níveis de LDL-C, TC e IL-6 nos grupos experimentais, particularmente na dose de $50 \mathrm{mg} / \mathrm{kg}$ de GSE, diminuíram significativamente em comparação com o grupo PCOS. De acordo com os achados deste estudo, pode-se concluir que o GSE com seus efeitos sobre a CT sérica, LDL-C e IL-6 poderia reduzir os efeitos da dislipidemia, melhorar os sintomas sistêmicos e inflamação em ratos com POCS.

Corroborando com estudos anteriores com doses de 50, 75, $100 \mathrm{mg} / \mathrm{kg}$ de GSE, os quais aumentaram, significativamente, o número de folículos priomordiais, folículos antrais e Graafianos, como também o número de corpo lúteo, indicando uma melhora considerável nos ovários policísticos (28). Com o GSE na dose de $200 \mathrm{mg} / \mathrm{kg}$ ocorreu inflamação visceral notável, acúmulo de líquido na cavidade peritoneal e danos graves a vários órgãos (principalmente o fígado), portanto, o GSE nesta dose foi considerado tóxico. Contudo, as duas doses de 50 e $75 \mathrm{mg} / \mathrm{kg}$ de GSE, devido aos seus efeitos melhoradores sobre os sintomas sistêmicos da POCS, foram consideradas doses efetivas (29). Com a melhora dos sintomas da POCS, ocorre também um efeito benéfico quanto a fertilidade de ratas suplementadas com extrato de semente de uva em proporções não toxicas.

Petrolini A. Viabilidade ovariana com o uso do extrato de semente de uva: Uma Revisão Sistemática. Vet. e Zootec. 2021; v28: 001-009. 


\section{CONCLUSÃO}

Essa revisão sistemática demonstrou achados de estudos sobre a utilização do extrato de semente de uva, o qual pode ser benéfico para a viabilidade da saúde ovariana, pois causa homeostase a respeito do estresse oxidativo, ocasionando uma possível reprodução viável, no entanto, faz-se necessário estudos mais aprofundados sobre o assunto.

Dessa maneira, observa-se a necessidade de pesquisas experimentais e ensaios clínicos com maior número de amostras para reforçar as evidências de que o extrato de semente de uva é uma terapia benéfica e segura no combate e prevenção de complicações ovarianas, bem como, o aproveitamento dos resíduos gerados pela agroindústria da uva.

\section{REFERÊNCIAS}

1. Kobori CN, Jorge N. Characterization of some seed oils of fruits for utilization of industrial residues. Cienc Agrotec. 2005;29(5):1008-14.

2. Kim Y, Choi Y, Ham H, Jeong HS, Lee J. Protective effects of oligomeric and polymeric procyanidin fractions from defatted grape seeds on tert-butyl hydroperoxide-induced oxidative damage in HepG2 cells. Food Chem. 2013;137(1-4):136-41. doi: http://dx.doi.org/10.1016/j.foodchem.2012.10.006.

3. Peralbo-Molina Á, Luque deCastro MD. Potential of residues from the Mediterranean agriculture and agrifood industry. Trends Food Sci Technol. 2013;32(1):16-24.

4. Puiggròs F, Llópiz N, Ardévol A, Bladé C, Arola L, Salvadó MJ. Grape seed procyanidins prevent oxidative injury by modulating the expression of antioxidant enzyme systems. $\mathrm{J}$ Agric Food Chem. 2005;53(15):6080-6.

5. Guo L, Li HW, Sun B, Jing YY, Yu QZ, Ying XD, et al. Direct in vivo evidence of protective effects of grape seed procyanidin fractions and other antioxidants against ethanol-induced oxidative DNA damage in mouse brain cells. J Agric Food Chem. 2007;55(14):5881-91.

6. Vinson JA, Mandarano MA, Shuta DL, Bagchi M, Bagchi D. Beneficial effects of a novel IH636 grape seed proanthocyanidin extract and a niacin-bound chromium in a hamster atherosclerosis model. Mol Cell Biochem. 2002;240(1-2):99-103.

7. Mantena SK, Baliga MS, Katiyar SK. Grape seed proanthocyanidins induce apoptosis and inhibit metastasis of highly metastatic breast carcinoma cells. Carcinogenesis. 2006;27(8):1682-91.

8. Wang YJ, Thomas P, Zhong JH, Bi FF, Kosaraju S, Pollard A, et al. Consumption of grape seed extract prevents amyloid- $\beta$ deposition and attenuates inflammation in brain of an alzheimer's disease mouse. Neurotox Res. 2009;15(1):3-14.

9. Ghafoor K, Choi YH, Jeon JY, Jo IH. Optimization of ultrasound-assisted extraction of phenolic compounds, antioxidants, and anthocyanins from grape (Vitis vinifera) seeds. $\mathrm{J}$ Agric Food Chem. 2009;57(11):4988-94. 
10. Campos LMAS, Leimann FV, Pedrosa RC, Ferreira SRS. Free radical scavenging of grape pomace extracts from Cabernet sauvingnon (Vitis vinifera). Bioresour Technol. 2008;99(17):8413-20.

11. García-Lomillo J, González-SanJosé ML, Del Pino-García R, Rivero-Pérez MD, MuñizRodríguez P. Antioxidant and antimicrobial properties of wine by products and their potential uses in the food industry. J Agric Food Chem. 2014;62(52):12595-602.

12. Chidambara Murthy KN, Singh RP, Jayaprakasha GK. Antioxidant activities of grape (Vitis vinifera) pomace extracts. J Agric Food Chem. 2002;50(21):5909-14.

13. Lachman S, Peters RJG, Lentjes MAH, Mulligan AA, Luben RN, Wareham NJ, et al. Ideal cardiovascular health and risk of cardiovascular events in the EPIC-Norfolk prospective population study. Eur J Prev Cardiol. 2015;23(9):986-94.

14. Vuong TV, Franco C, Zhang W. Treatment strategies for high resveratrol induction in Vitis vinifera L. cell suspension culture. Biotechnol Rep. 2014;1-2:15-21. doi: http://dx.doi.org/10.1016/j.btre.2014.04.002.

15. Bezerra MÉS, Gouveia BB, Barberino RS, Menezes VG, Macedo TJS, Cavalcante AYP, et al. Resveratrol promotes in vitro activation of ovine primordial follicles by reducing DNA damage and enhancing granulosa cell proliferation via phosphatidylinositol 3kinase pathway. Reprod Domest Anim. 2018;53(6):1298-305.

16. Sosa V, Moliné T, Somoza R, Paciucci R, Kondoh H, LLeonart ME. Oxidative stress and cancer: an overview. Ageing Res Rev. 2013;12(1):376-90. doi: http://dx.doi.org/10.1016/j.arr.2012.10.004.

17. Rodgers RJ. Steroidogenic cytochrome p450 enzymes and ovarian steroidogenesis. Reprod Fertil Dev. 1990;2(2):153-63.

18. Imai H, Nakagawa Y. Regulatory and Cytoprotective Aspects of Lipid Hydroperoxide Metabolism. Free Radic Biol Med. 2003;34(2):145-69.

19. Zhang JQ, Xing BS, Zhu CC, Shen M, Yu FX, Liu HL. Protective effect of proanthocyanidin against oxidative ovarian damage induced by 3-nitropropionic acid in mice. Genet Mol Res. 2015;14(1):2484-94.

20. Bao L, Cai X, Zhang Z, Li Y. Grape seed procyanidin B2 ameliorates mitochondrial dysfunction and inhibits apoptosis via the AMP-Activated protein kinase-silent mating type information regulation 2 homologue 1-PPAR $\gamma$ co-Activator-1 $\alpha$ axis in rat mesangial cells under high-dose glucosami. Br J Nutr. 2015;113(1):35-44.

21. Long M, Liu Y, Cao Y, Wang N, Dang M, He J. Proanthocyanidins attenuation of chronic lead-induced liver oxidative damage in kunming mice via the Nrf2/ARE pathway. Nutrients. 2016;8(10):656.

22. Zhang JQ, Gao BW, Wang J, Ren QL, Chen JF, Ma Q, et al. Critical role of foxo1 in granulosa cell apoptosis caused by oxidative stress and protective effects of grape seed procyanidin B2. Oxid Med Cell Longev. 2016;2016:6147345. 
23. Yang H, Xiao L, Yuan Y, Luo X, Jiang M, Ni J, et al. Procyanidin B2 inhibits NLRP3 inflammasome activation in human vascular endothelial cells. Biochem Pharmacol. 2014;92(4):599-606. doi: http://dx.doi.org/10.1016/j.bcp.2014.10.001.

24. Zhang JQ, Wang XW, Chen JF, Ren QL, Wang J, Gao BW, et al. Grape seed Procyanidin B2 protects porcine ovarian granulosa cells against oxidative stress-induced apoptosis by upregulating let-7a expression. Oxid Med Cell Longev. 2019;2019:1076512.

25. Wang X, Zhu X, Liang X, Xu H, Liao Y, Lu K, et al. Effects of resveratrol on in vitro maturation of porcine oocytes and subsequent early embryonic development following somatic cell nuclear transfer. Reprod Domest Anim. 2019;54(9):1195-205.

26. Liu X, Lin X, Mi Y, Li J, Zhang C. Grape seed Proanthocyanidin extract prevents ovarian aging by inhibiting oxidative stress in the hens. Oxid Med Cell Longev. 2018;2018:9390810.

27. Salmabadi Z, Kouchesfahani HM, Parivar K, Karimzadeh L. Effect of grape seed extract on lipid profile and expression of lnterleukin-6 in polycystic ovarian syndrome wistar rat model. Int J Fertil Steril. 2017;11(3):176-83.

28. Mohseni KH, Parivar K, Salmabadi Z. Effect of hydroalcoholic grape seed extract (Vitis vinifera L.) on polycystic ovary syndrome in female Wistar rats. J Cell Tissue. 2015;6(2):153-65.

29. Sato M, Bagchi D, Tosaki A, Das DK. Grape seed proanthocyanidin reduces cardiomyocyte apoptosis by inhibiting ischemia/reperfusion-induced activation of JNK-1 and C-JUN. Free Radic Biol Med. 2001;31(6):729-37.

Recebido em: 29/06/2020

Aceito em: 22/04/2021 